\title{
ASYMPTOTIC BEHAVIOR OF CONTINUOUS TRAJECTORIES FOR PRIMAL-DUAL POTENTIAL-REDUCTION METHODS
}

\author{
REHA H. TÜTÜNCÜ*
}

\begin{abstract}
This article considers continuous trajectories of the vector fields induced by primaldual potential-reduction algorithms for solving linear programming problems. It is known that these trajectories converge to the analytic center of the primal-dual optimal face. We establish that this convergence may be tangential to the central path, tangential to the optimal face, or in between, depending on the value of the potential function parameter.
\end{abstract}

Key words. linear programming, potential functions, potential-reduction methods, central path, continuous trajectories for linear programming.

AMS subject classifications. 90C05

1. Introduction. During the past two decades, interior-point methods (IPMs) emerged as one of the most efficient and reliable techniques for the solution of linear programming problems. The development of IPMs and their theoretical convergence analyses often rely on certain continuous trajectories associated with the given linear program. The best known examples of such trajectories are the central path and the weighted centers - the sets of minimizers of the parametrized standard and weighted logarithmic barrier functions in the interior of the feasible region.

Primal-dual variants of IPMs, which have been very successful in practical implementations, not only solve the given linear program but also its dual. If both the given LP and its dual have strictly feasible solutions the primal-dual central path starts from the analytic center of the primal-dual feasible set and converges to the analytic center of the optimal solution set. Similarly, weighted centers converge to weighted analytic centers. This property of the central trajectories led to the development of path following IPMs: algorithms that try to reach an optimal solution by generating a sequence of points that are "close" to a corresponding sequence of points on the central path (or the weighted central path) that converge to its limit point.

An alternative characterization of the central path and weighted centers can be obtained by representing them as solutions of certain differential equations. Using this perspective, Adler and Monteiro analyzed the limiting behavior of continuous trajectories associated with primal-only affine-scaling and projective-scaling algorithms as well as a primal-only potential-reduction method $[1,11,12]$. Kojima et al. studied similar trajectories for primal-dual potential-reduction methods [7].

Potential-reduction algorithms use the following strategy: First, one defines a potential function that measures the quality (or potential) of any trial solution of the given problem combining measures of proximity to the set of optimal solutions, proximity to the feasible set in the case of infeasible-interior-points, and a measure of centrality within the feasible region. Potential functions are chosen such that one approaches an optimal solution of the underlying problem by reducing the potential function. Then, the search for an optimal solution can be performed by progressive reduction of the potential function, leading to a potential-reduction algorithm. We refer the reader to two excellent surveys for further details on potential-reduction algorithms $[2,15]$.

${ }^{*}$ Department of Mathematical Sciences, Carnegie Mellon University, Pittsburgh, PA 15213, USA (reha@cmu.edu). Research supported in part by NSF through grant CCR-9875559. 
Often, implementations of potential-reduction interior-point algorithms exhibit behavior that is similar to that of path-following algorithms. For example, they take about the same number of iterations as path-following algorithms and they tend to converge to the analytic center of the optimal face, just like most path-following variants. Since potential-reduction methods do not generally make an effort to follow the central path, this behavior is surprising. In an effort to better understand the limiting behavior of primal-dual potential-reduction algorithms for linear programs this paper studies continuous trajectories associated with the algorithm proposed by Kojima, Mizuno, and Yoshise (KMY) [8], which uses scaled and projected steepest descent directions for the Tanabe-Todd-Ye (TTY) primal-dual potential function [14, $16]$.

Using earlier results $[9,10,7]$, we show that all trajectories of the vector field induced by the KMY search directions converge to the analytic center of the primaldual optimal face. Our main results are on the direction of convergence for these trajectories. We demonstrate that their asymptotic behavior depends on the potential function parameter. There is a threshold value of this parameter-the value that makes the TTY potential function homogeneous. When the parameter is below this threshold, the centering is too strong and the trajectories converge tangentially to the central path. When the parameter is above the threshold, trajectories converge tangentially to the optimal face. However, the direction of convergence of these trajectories depends on the initial point. At the threshold value, the behavior of the trajectories is in between these two extremes and depends on the initial point.

Following this introduction, Section 2 discusses continuous trajectories associated with the KMY methods and proves their convergence. Section 3 is devoted to the analysis of the limiting behavior of these trajectories. Our notation is fairly standard: For an $n$-dimensional vector $x$, the corresponding capital letter $X$ denotes the $n \times n$ diagonal matrix with $X_{i i} \equiv x_{i}$. We will use the letter $e$ to denote a column vector with all entries equal to 1 and its dimension will be apparent from the context. We also denote the base of the natural logarithm with $e$ and sometimes the vector $e$ and the scalar $e$ appear in the same expression, but no confusion should arise. For a given matrix $A$, we use $\mathcal{R}(A)$ and $\mathcal{N}(A)$ to denote its range(column) and null space. For a vector-valued differentiable function $x(t)$ of a scalar variable $t$, we use the notation $\dot{x}$ or $\dot{x}(t)$ to denote the vector of the derivatives of its components with respect to $t$. For $n$ dimensional vectors $x$ and $s$, we write $x s$ to denote their Hadamard (componentwise) product. Also, for an $n$ dimensional vector $x$, we write $x^{p}$ to denote the vector $X^{p} e$, where $p$ can be fractional if $x>0$.

2. Primal-Dual Potential-Reduction Trajectories. We consider linear programs in the following standard form:

$$
\begin{array}{r}
\min _{x} c^{T} x \\
A x=b \\
x \geq 0,
\end{array}
$$

where $A \in \Re^{m \times n}, b \in \Re^{m}, c \in \Re^{n}$ are given, and $x \in \Re^{n}$. Without loss of generality we assume that the constraints are linearly independent. Then, the matrix $A$ has full row rank. Further, we assume that $0<m<n ; m=0$ and $m=n$ correspond to trivial problems. 
The linear programming dual of this (primal) problem is:

$$
\begin{aligned}
&(L D) \max _{y, s} b^{T} y \\
& A^{T} y+s=c \\
& s \geq 0,
\end{aligned}
$$

where $y \in \Re^{m}$ and $s \in \Re^{n}$. We can rewrite the dual problem by eliminating the $y$ variables in (2.2). This is achieved by considering $G^{T}$, a null-space basis matrix for $A$, that is, $G$ is an $(n-m) \times n$ matrix with rank $n-m$ and it satisfies $A G^{T}=0$, $G A^{T}=0$. Note also that, $A^{T}$ is a null-space basis matrix for $G$. Further, let $d \in \Re^{n}$ be a vector satisfying $A d=b$. Then, (2.2) is equivalent to the following problem which has a high degree of symmetry with (2.1):

$$
\begin{aligned}
\left(L D^{\prime}\right) \quad \min _{s} d^{T} s & \\
G s & =G c \\
s & \geq 0 .
\end{aligned}
$$

Let $\mathcal{F}$ and $\mathcal{F}^{0}$ denote the primal-dual feasible region and its relative interior:

$$
\begin{aligned}
\mathcal{F} & :=\{(x, s): A x=b, G s=G c,(x, s) \geq 0\}, \\
\mathcal{F}^{0} & :=\{(x, s): A x=b, G s=G c,(x, s)>0\} .
\end{aligned}
$$

We assume that $\mathcal{F}^{0}$ is non-empty. This assumption has the important consequence that the primal-dual optimal solution set $\Omega$ defined below is nonempty and bounded:

$$
\Omega:=\left\{(x, s) \in \mathcal{F}: x^{T} s=0\right\} .
$$

We also define the optimal partition $\mathcal{B} \cup \mathcal{N}=\{1, \ldots, n\}$ for future reference:

$$
\begin{aligned}
& \mathcal{B}:=\left\{j: x_{j}>0 \text { for some }(x, s) \in \Omega\right\} \\
& \mathcal{N}:=\left\{j: s_{j}>0 \text { for some }(x, s) \in \Omega\right\} .
\end{aligned}
$$

The fact that $\mathcal{B}$ and $\mathcal{N}$ is a partition of $\{1, \ldots, n\}$ is a classical result of Goldman and Tucker. The analytic center of $\Omega$ is the point $\left(x^{*}, s^{*}\right)=\left(\left(x_{\mathcal{B}}^{*}, 0\right),\left(0, s_{\mathcal{N}}^{*}\right)\right)$ where $x_{\mathcal{B}}^{*}$ and $s_{\mathcal{N}}^{*}$ are unique maximizers of the following problems:

$$
\begin{array}{ccc}
\max & \begin{array}{c}
\sum_{j \in \mathcal{B}} \ln x_{j} \\
A_{\mathcal{B}} x_{\mathcal{B}}=b \quad \text { max }
\end{array} & \sum_{j \in \mathcal{N}} \ln s_{j} \\
x_{\mathcal{B}}>0, & & G_{\mathcal{N}} s_{\mathcal{N}}=G c \\
& & s_{\mathcal{N}}>0
\end{array}
$$

The central path $\mathcal{C}$ of the primal-dual feasible set $\mathcal{F}$ is the set of points on which the component-wise product of the primal and dual variables is constant:

$$
\mathcal{C}:=\left\{(x(\mu), s(\mu)) \in \mathcal{F}^{0}: x(\mu) s(\mu)=\mu e, \text { for some } \mu>0\right\} .
$$

The points on the central path are obtained as unique minimizers of certain barrier problems associated with the primal and dual LPs and they converge to the analytic center of the primal-dual optimal face; see, e.g., [18].

While the central path is the main theoretical tool in the construction of pathfollowing algorithms, primal-dual potential-reduction algorithms for linear programming are derived using potential functions, i.e., functions that measure the quality 
(or potential) of trial solutions for the primal-dual pair of problems. The most frequently used primal-dual potential function for linear programming problems is the Tanabe-Todd-Ye (TTY) potential function $[14,16]$ :

$$
\Phi_{\rho}(x, s):=\rho \ln \left(x^{T} s\right)-\sum_{i=1}^{n} \ln \left(x_{i} s_{i}\right), \text { for every }(x, s)>0 .
$$

When $\rho>n$, the TTY potential function diverges to $-\infty$ along a feasible sequence $\left\{\left(x^{k}, s^{k}\right)\right\}$ only if this sequence is converging to a primal-dual optimal pair of solutions. Therefore, the primal-dual pair of LP problems can be solved by minimizing the TTY potential function.

Kojima, Mizuno, and Yoshise (KMY) developed a primal-dual algorithm that monotonically reduces the TTY potential function using a scaled and projected steepestdescent search direction using a primal-dual scaling matrix [8]. In the remainder of this article, we will study continuous trajectories that are naturally associated with their algorithm. Given an iterate $(x, s) \in \mathcal{F}^{0}$, the search direction used by the KMY method is the solution of the following system:

$$
\begin{aligned}
A \Delta x & =0 \\
G \Delta s & =0 \\
S \Delta x+X \Delta s & =\frac{x^{T} s}{\rho} e-x s,
\end{aligned}
$$

where $X=\operatorname{diag}(x), S=\operatorname{diag}(s)$, and $e$ is a vector of ones of appropriate dimension. When we discuss the search direction given by (2.8) and associated trajectories, we will assume that $\rho>n$.

For any given $\left(x_{0}, s_{0}\right) \in \mathcal{F}^{0}$, one can associate a trajectory $\{(x(t), s(t)): t \geq 0\}$ starting from $\left(x_{0}, s_{0}\right)$ with the property that the tangent direction to the trajectory at any of its points coincides with the KMY direction. In other words, we consider trajectories that solve the following system of ordinary differential equations:

$$
\left[\begin{array}{cc}
A & 0 \\
0 & G \\
S & X
\end{array}\right]\left[\begin{array}{c}
\dot{x} \\
\dot{s}
\end{array}\right]=\left[\begin{array}{c}
0 \\
0 \\
\frac{x^{T} s}{\rho} e-x s
\end{array}\right],
$$

with the initial condition $(x(0), s(0))=\left(x_{0}, s_{0}\right)$. In [7, Section 4.3], Kojima et al. study these trajectories and establish that their solution curves satisfy the following system of equations:

$$
A x(t)=b, G s(t)=G c, x(t) s(t)=w(t), t \geq 0,
$$

where

$$
\begin{gathered}
w(t)=e^{-t} w_{0}+h(t) e, \text { with } w_{0}=x_{0} s_{0}, \text { and, } \\
h(t)=\frac{e^{T} w_{0}}{n}\left(\exp \{-(1-\beta) t\}-e^{-t}\right) \text { with } \beta=\frac{n}{\rho} .
\end{gathered}
$$

Since $w(0)=w_{0}$, we will use these two expressions interchangeably. Kojima et al. do not address the existence and uniqueness of the solutions to (2.9) rigorously, but these results follow easily from standard theory of ordinary differential equations; see, 
e.g., Theorem 1 on p. 162 and Lemma on p. 171 of the textbook by Hirsch and Smale [6]. We also note that Monteiro [12] studies trajectories based on primal-only potential-reduction algorithms and obtains similar but less explicit descriptions of these trajectories.

The characterization of the potential-reduction trajectories using the system (2.10) leads to the following observations:

ThEOREM 2.1. Let $(x(t), s(t))$ for $t \geq 0$ denote the solution of the ODE (2.9) with the initial condition $(x(0), s(0))=\left(x_{0}, s_{0}\right)$. Then, the following statements hold:

(i) For $\rho>n, \Phi_{\rho}(x(t), s(t))$ is a decreasing function of $t$.

(ii) When $w_{0}=x_{0} s_{0}=\mu e$ for some $\mu>0$ (i.e., when $\left(x_{0}, s_{0}\right)$ is on the central path) then $\{(x(t), s(t)): t \geq 0\}$ is a subset of the central path $\mathcal{C}$.

(iii) $(x(t), s(t))$ converges to the analytic center of the primal-dual optimal face $\Omega$ as $t \rightarrow \infty$.

Proof. Lemma 4.14 in [7] proves (i). Observing that $w_{0}=\mu e$ implies $w(t)=$ $\mu e^{-(1-\beta) t} e$ with $\beta=\frac{n}{\rho}$, (ii) follows immediately from (2.6) and (2.10). For (iii), first observe that $\frac{w(t)}{\|w(t)\|} \rightarrow \frac{e}{\sqrt{n}}$ as $t \rightarrow \infty$. Now, the proof of Theorem 9 in [9] (or, Corollary 2 of Theorem 5 in [10]) immediately leads to (iii).

So, these trajectories converge to a unique point regardless of their starting point as they monotonically decrease the potential function. Further, they include the central path as a special case giving a theoretical basis for the observation that central path-following search directions are often very good potential-reduction directions as well. Another related result is by Nesterov [13] who observes that the neighborhood of the central path is the region of fastest decrease for a homogeneous potential function.

A direct proof of (iii) in Theorem 2.1 can be obtained using the following result, which will also be useful in the next section:

Lemma 2.2. Let $(x(t), s(t))$ for $t \geq 0$ denote the solution of the ODE (2.9) with the initial condition $(x(0), s(0))=\left(x_{0}, s_{0}\right)$. Then $x_{\mathcal{B}}(t)$ and $s_{\mathcal{N}}(t)$ solve the following pair of problems:

$$
\begin{aligned}
& \max \quad \sum_{j \in \mathcal{B}} w_{j}(t) \ln x_{j} \quad \max \quad \sum_{j \in \mathcal{N}} w_{j}(t) \ln s_{j} \\
& A_{\mathcal{B}} x_{\mathcal{B}}=b-A_{\mathcal{N}} x_{\mathcal{N}}(t) \text { and } \quad G_{\mathcal{N}} s_{\mathcal{N}}=G c-G_{\mathcal{B}} s_{\mathcal{B}}(t) \\
& x_{\mathcal{B}}>0, \quad s_{\mathcal{N}}>0 .
\end{aligned}
$$

Proof. We prove the optimality of $x_{\mathcal{B}}(t)$ for the first problem in (2.13)-the corresponding result for $s_{\mathcal{N}}(t)$ can be proven similarly. $x_{\mathcal{B}}(t)$ is clearly feasible for the given problem. It is optimal if and only if there exists $y \in \Re^{m}$ such that

$$
w_{\mathcal{B}}(t) x_{\mathcal{B}}^{-1}(t)=A_{\mathcal{B}}^{T} y \text {. }
$$

From (2.10) we obtain $w_{\mathcal{B}}(t) x_{\mathcal{B}}^{-1}(t)=s_{\mathcal{B}}(t)$. Note that, for any $s$ feasible for (LD') we have that $c-s \in \mathcal{R}\left(A^{T}\right)$ and therefore, $c_{\mathcal{B}}-s_{\mathcal{B}} \in \mathcal{R}\left(A_{\mathcal{B}}^{T}\right)$. Furthermore, since $s^{*}=\left(0, s_{\mathcal{N}}^{*}\right)$ is also feasible for (LD') we must have that $c_{\mathcal{B}} \in \mathcal{R}\left(A_{\mathcal{B}}^{T}\right)$ and that $s_{\mathcal{B}}(t) \in \mathcal{R}\left(A_{\mathcal{B}}^{T}\right)$. This is exactly what we needed.

3. Asymptotic Analysis of the Trajectories. In the previous section, we saw that all primal-dual potential-reduction trajectories $(x(t), s(t))$ that solve the differential equation (2.9) converge to the analytic center $\left(x^{*}, s^{*}\right)$ of the primal-dual optimal face $\Omega$ regardless of the initial point of the trajectory. In this section, we investigate the direction of convergence for these trajectories. That is, we want to analyze the limiting behavior of the normalized vectors $\left(\frac{\dot{x}(t)}{\|\dot{x}(t)\|}, \frac{\dot{s}(t)}{\|\dot{s}(t)\|}\right)$. Inevitably, this analysis is quite technical. 
Our strategy for this analysis is as follows. Using the optimal partition $\mathcal{B}$ and $\mathcal{N}$ we express the "basic" components of the convergence directions of the trajectories in terms of the "nonbasic" ones in Lemma 3.1. Then, we establish a bound on the convergence speed of the "nonbasic" components in Lemma 3.3. The dependence of the convergence direction on $\rho$, the potential function parameter, becomes apparent at this point and a case analysis is required. Lemma 3.4, Theorems 3.5 and 3.6 consider the case $\rho \leq 2 n$ while Theorem 3.8 addresses the case $\rho>2 n$.

Let $\beta=\frac{n}{\rho}$ and note that $\beta \in(0,1)$. We now introduce some notation:

$$
\begin{array}{ll}
\hat{w}_{\mathcal{B}}(t)=w_{\mathcal{B}}(t) e^{(1-\beta) t}, & \hat{w}_{\mathcal{N}}(t)=w_{\mathcal{N}}(t) e^{(1-\beta) t}, \\
d_{\mathcal{B}}(t)=\hat{w}_{\mathcal{B}}^{\frac{1}{2}}(t) x_{\mathcal{B}}^{-1}(t), & d_{\mathcal{N}}(t)=\hat{w}_{\mathcal{N}}^{\frac{1}{2}}(t) s_{\mathcal{N}}^{-1}(t), \\
D_{\mathcal{B}}(t)=\operatorname{diag}\left(d_{\mathcal{B}}(t)\right), & D_{\mathcal{N}}(t)=\operatorname{diag}\left(d_{\mathcal{N}}(t)\right), \\
d_{\mathcal{B}}^{-1}(t)=D_{\mathcal{B}}^{-1}(t) e, & d_{\mathcal{N}}^{-1}(t)=D_{\mathcal{N}}^{-1}(t) e, \\
\tilde{A}_{\mathcal{B}}(t)=A_{\mathcal{B}} D_{\mathcal{B}}^{-1}(t), & \tilde{\widetilde{G}}_{\mathcal{N}}(t)=G_{\mathcal{N}} D_{\mathcal{\mathcal { N }}}^{-1}(t), \\
\tilde{x}_{\mathcal{B}}(t)=D_{\mathcal{B}}(t) \dot{x}_{\mathcal{B}}(t)=d_{\mathcal{B}}(t) \dot{x}_{\mathcal{B}}(t), & \tilde{s}_{\mathcal{N}}(t)=D_{\mathcal{N}}(t) \dot{s}_{\mathcal{N}}(t)=d_{\mathcal{N}}(t) \dot{s}_{\mathcal{N}}(t), \\
u_{\mathcal{B}}(t)=\hat{w}_{\mathcal{B}}^{-\frac{1}{2}}(t) w_{\mathcal{B}}(0), & u_{\mathcal{N}}(t)=\hat{w}_{\mathcal{N}}^{-\frac{1}{2}}(t) w_{\mathcal{N}}(0) .
\end{array}
$$

For our asymptotic analysis, we express "basic" components of the vectors $\dot{x}(t)$ and $\dot{s}(t)$ in terms of the "nonbasic" ones in the next lemma which forms the backbone of our analysis.

LEMma 3.1. Let $(x(t), s(t))$ for $t \geq 0$ denote the solution of the ODE (2.9) with the initial condition $(x(0), s(0))=\left(x_{0}, s_{0}\right)$. Then, the following equalities hold:

$$
\begin{aligned}
& D_{\mathcal{B}}(t) \dot{x}_{\mathcal{B}}(t)=-\tilde{A}_{\mathcal{B}}^{+}(t) A_{\mathcal{N}} \dot{x}_{\mathcal{N}}(t)-\frac{n \cdot e^{-\beta t}}{\rho\left(1-e^{-\beta t}\right)}\left(I-\tilde{A}_{\mathcal{B}}^{+}(t) \tilde{A}_{\mathcal{B}}(t)\right) u_{\mathcal{B}}(t), \\
& D_{\mathcal{N}}(t) \dot{s}_{\mathcal{N}}(t)=-\tilde{G}_{\mathcal{N}}^{+}(t) G_{\mathcal{B}} \dot{s}_{\mathcal{B}}(t)-\frac{n \cdot e^{-\beta t}}{\rho\left(1-e^{-\beta t}\right)}\left(I-\tilde{G}_{\mathcal{N}}^{+}(t) \tilde{G}_{\mathcal{N}}(t)\right) u_{\mathcal{N}}(t)
\end{aligned}
$$

Here, $\tilde{A}_{\mathcal{B}}^{+}(t)$ and $\tilde{G}_{\mathcal{N}}^{+}(t)$ denote the pseudo-inverse of $\tilde{A}_{\mathcal{B}}(t)$ and $\tilde{G}_{\mathcal{N}}(t)$, respectively

Proof. We only prove the first identity; the second one follows similarly. Recall from Lemma 2.2 that $x_{\mathcal{B}}(t)$ solves the first problem in (2.13). Therefore, as in the proof of Lemma 2.2, we must have that

$$
w_{\mathcal{B}}(t) x_{\mathcal{B}}^{-1}(t) \in \mathcal{R}\left(A_{\mathcal{B}}^{T}\right)
$$

Differentiating with respect to $t$ we obtain:

$$
\begin{aligned}
& w_{\mathcal{B}}(t) x_{\mathcal{B}}^{-2}(t) \dot{x}_{\mathcal{B}}(t)-\dot{w}_{\mathcal{B}}(t) x_{\mathcal{B}}^{-1}(t) \in \mathcal{R}\left(A_{\mathcal{B}}^{T}\right), \text { or } \\
& w_{\mathcal{B}}(t) x_{\mathcal{B}}^{-1}(t) \dot{x}_{\mathcal{B}}(t)-\dot{w}_{\mathcal{B}}(t) \in \mathcal{R}\left(X_{\mathcal{B}}(t) A_{\mathcal{B}}^{T}\right) .
\end{aligned}
$$

Observe that

$$
\dot{w}_{\mathcal{B}}(t)=-w_{\mathcal{B}}(0) e^{-t}+\dot{h}(t) e_{\mathcal{B}}=-w_{\mathcal{B}}(t)+\frac{e^{T} w_{0}}{\rho} e^{-(1-\beta) t} e_{\mathcal{B}}
$$

Therefore, from $\hat{w}_{\mathcal{B}}(t)=w_{\mathcal{B}}(t) e^{(1-\beta) t}$, we obtain

$$
\hat{w}_{\mathcal{B}}(t) x_{\mathcal{B}}^{-1}(t) \dot{x}_{\mathcal{B}}(t)+\hat{w}_{\mathcal{B}}(t)-\frac{e^{T} w_{0}}{\rho} e_{\mathcal{B}} \in \mathcal{R}\left(X_{\mathcal{B}}(t) A_{\mathcal{B}}^{T}\right)
$$


From (3.3) it also follows that $\hat{w}_{\mathcal{B}}(t) \in \mathcal{R}\left(X_{\mathcal{B}}(t) A_{\mathcal{B}}^{T}\right)$. Note also that,

$$
\frac{e^{T} w_{0}}{\rho} e_{\mathcal{B}}=\frac{n}{\rho\left(1-e^{-\beta t}\right)} \hat{w}_{\mathcal{B}}(t)-\frac{n \cdot e^{-\beta t}}{\rho\left(1-e^{-\beta t}\right)} w_{\mathcal{B}}(0) .
$$

Combining these observations with (3.4) we get

$$
\hat{w}_{\mathcal{B}}(t) x_{\mathcal{B}}^{-1}(t) \dot{x}_{\mathcal{B}}(t)+\frac{n \cdot e^{-\beta t}}{\rho\left(1-e^{-\beta t}\right)} w_{\mathcal{B}}(0) \in \mathcal{R}\left(X_{\mathcal{B}}(t) A_{\mathcal{B}}^{T}\right) .
$$

Next, observe that

$$
A_{\mathcal{B}} \dot{x}_{\mathcal{B}}(t)=-A_{\mathcal{N}} \dot{x}_{\mathcal{N}}(t) .
$$

Using the notation introduced before the statement of the lemma, (3.5) and (3.6) can be rewritten as follows:

$$
\begin{gathered}
\tilde{x}_{\mathcal{B}}(t)+\frac{n \cdot e^{-\beta t}}{\rho\left(1-e^{-\beta t}\right)} u_{\mathcal{B}}(t) \in \mathcal{R}\left(\tilde{A}_{\mathcal{B}}^{T}\right), \\
\tilde{A}_{\mathcal{B}}(t) \tilde{x}_{\mathcal{B}}(t)=-A_{\mathcal{N}} \dot{x}_{\mathcal{N}}(t) .
\end{gathered}
$$

Let $\tilde{A}_{\mathcal{B}}^{+}(t)$ denote the pseudo-inverse of $\tilde{A}_{\mathcal{B}}(t)$ [3]. For example, if $\operatorname{rank}\left(\tilde{A}_{\mathcal{B}}(t)\right)=m$, then $\tilde{A}_{\mathcal{B}}^{+}(t)=\tilde{A}_{\mathcal{B}}^{T}(t)\left(\tilde{A}_{\mathcal{B}}(t) \tilde{A}_{\mathcal{B}}^{T}(t)\right)^{-1}$. Then, $P_{\mathcal{R}\left(\tilde{A}_{\mathcal{B}}^{T}\right)}:=\tilde{A}_{\mathcal{B}}^{+}(t) \tilde{A}_{\mathcal{B}}(t)$ is the orthogonal projection matrix onto $\mathcal{R}\left(\tilde{A}_{\mathcal{B}}^{T}\right)$ and $P_{\mathcal{N}\left(\tilde{A}_{\mathcal{B}}\right)}:=I-\tilde{A}_{\mathcal{B}}^{+}(t) \tilde{A}_{\mathcal{B}}(t)$ is the orthogonal projection matrix onto $\mathcal{N}\left(\tilde{A}_{\mathcal{B}}\right)[3]$. From (3.8) we obtain

$$
P_{\mathcal{R}\left(\tilde{A}_{\mathcal{B}}^{T}\right)} \tilde{x}_{\mathcal{B}}(t)=\tilde{A}_{\mathcal{B}}^{+}(t) \tilde{A}_{\mathcal{B}}(t) \tilde{x}_{\mathcal{B}}(t)=-\tilde{A}_{\mathcal{B}}^{+}(t) A_{\mathcal{N}} \dot{x}_{\mathcal{N}}(t)
$$

and from (3.7), using the fact that $\mathcal{R}\left(\tilde{A}_{\mathcal{B}}^{T}\right)$ and $\mathcal{N}\left(\tilde{A}_{\mathcal{B}}\right)$ are orthogonal to each other, we get

$$
P_{\mathcal{N}\left(\tilde{A}_{\mathcal{B}}\right)} \tilde{x}_{\mathcal{B}}(t)=-\frac{n \cdot e^{-\beta t}}{\rho\left(1-e^{-\beta t}\right)}\left(I-\tilde{A}_{\mathcal{B}}^{+}(t) \tilde{A}_{\mathcal{B}}(t)\right) u_{\mathcal{B}}(t)
$$

Combining, we have

$$
\begin{aligned}
\tilde{x}_{\mathcal{B}}(t) & =P_{\mathcal{R}\left(\tilde{A}_{\mathcal{B}}^{T}\right)} \tilde{x}_{\mathcal{B}}(t)+P_{\mathcal{N}\left(\tilde{A}_{\mathcal{B}}\right)} \tilde{x}_{\mathcal{B}}(t) \\
& =-\tilde{A}_{\mathcal{B}}^{+}(t) A_{\mathcal{N}} \dot{x}_{\mathcal{N}}(t)-\frac{n \cdot e^{-\beta t}}{\rho\left(1-e^{-\beta t}\right)}\left(I-\tilde{A}_{\mathcal{B}}^{+}(t) \tilde{A}_{\mathcal{B}}(t)\right) u_{\mathcal{B}}(t),
\end{aligned}
$$

which gives (3.1).

To determine the convergence directions of the trajectories, we need to study the relative convergence speeds of $\dot{x}_{\mathcal{B}}(t), \dot{x}_{\mathcal{N}}(t)$, etc. Thus, we compute limits of some of the expressions that appear in equations (3.1) and (3.2):

$$
\begin{aligned}
\lim _{t \rightarrow \infty} \hat{w}_{\mathcal{B}}(t)=\frac{e^{T} w_{0}}{n} e_{\mathcal{B}}, & \lim _{t \rightarrow \infty} \hat{w}_{\mathcal{N}}(t)=\frac{e^{T} w_{0}}{n} e_{\mathcal{N}}, \\
\lim _{t \rightarrow \infty} D_{\mathcal{B}}(t)=\sqrt{\frac{e^{T} w_{0}}{n}}\left(X_{\mathcal{B}}^{*}\right)^{-1}, & \lim _{t \rightarrow \infty} D_{\mathcal{N}}(t)=\sqrt{\frac{e^{T} w_{0}}{n}}\left(S_{\mathcal{N}}^{*}\right)^{-1}, \\
\lim _{t \rightarrow \infty} \tilde{A}_{\mathcal{B}}(t)=\sqrt{\frac{n}{e^{T} w_{0}}} A_{\mathcal{B}} X_{\mathcal{B}}^{*}, & \lim _{t \rightarrow \infty} \tilde{G}_{\mathcal{N}}(t)=\sqrt{\frac{n}{e^{T} w_{0}}} G_{\mathcal{N}} S_{\mathcal{N}}^{*}, \\
\lim _{t \rightarrow \infty} u_{\mathcal{B}}(t)=\sqrt{\frac{n}{e^{T} w_{0}}} w_{\mathcal{B}}(0), & \lim _{t \rightarrow \infty} u_{\mathcal{N}}(t)=\sqrt{\frac{n}{e^{T} w_{0}}} w_{\mathcal{N}}(0) .
\end{aligned}
$$


LEMMA 3.2.

$$
\begin{aligned}
\lim _{t \rightarrow \infty} \tilde{A}_{\mathcal{B}}^{+}(t) & =\sqrt{\frac{e^{T} w_{0}}{n}}\left(A_{\mathcal{B}} X_{\mathcal{B}}^{*}\right)^{+} \\
\lim _{t \rightarrow \infty} \tilde{G}_{\mathcal{N}}^{+}(t) & =\sqrt{\frac{e^{T} w_{0}}{n}}\left(G_{\mathcal{N}} S_{\mathcal{N}}^{*}\right)^{+} .
\end{aligned}
$$

Proof. This result about the limiting properties of the pseudo-inverses is an immediate consequence of Lemma 2.3 in [4] and equations (3.11).

Differentiating the identity

$$
x(t) s(t)=w(t)=e^{-t} w_{0}+h(t) e,
$$

we obtain

$$
\begin{aligned}
x(t) \dot{s}(t)+\dot{x}(t) s(t) & =-e^{-t} w_{0}+\dot{h}(t) e \\
& =-e^{-t} w_{0}-\frac{e^{T} w_{0}}{n}(1-\beta) e^{-(1-\beta) t} e+\frac{e^{T} w_{0}}{n} e^{-t} e .
\end{aligned}
$$

Next, we will establish that $\dot{x}_{\mathcal{N}}(t)$ and $\dot{s}_{\mathcal{B}}(t)$ converge to zero no slower than $\exp \{-(1-$ $\beta) t\}$. For this purpose, we consider the normalized direction vectors $(\hat{x}, \hat{s})$ which are defined as follows:

$$
\hat{x}(t)=\exp \{(1-\beta) t\} \dot{x}(t), \text { and } \hat{s}(t)=\exp \{(1-\beta) t\} \dot{s}(t) .
$$

From (3.15) it follows that

$$
x(t) \hat{s}(t)+\hat{x}(t) s(t)=-\frac{e^{T} w_{0}}{n}(1-\beta) e+e^{-\beta t}\left(\frac{e^{T} w_{0}}{n} e-w_{0}\right) .
$$

The expression on the right-hand-side of (3.17) is clearly bounded. With some more work, we have the following conclusion:

LEMma 3.3. Let $(\hat{x}(t), \hat{s}(t))$ be as in (3.16) and assume that $\rho>n$. Then, $\left(\hat{x}_{\mathcal{N}}(t), \hat{s}_{\mathcal{B}}(t)\right)$ remains bounded as $t$ tends to $\infty$.

Proof. We will prove that $x(t) \hat{s}(t)$ and $\hat{x}(t) s(t)$ remain bounded as $t \rightarrow \infty$. Then, since $x_{\mathcal{B}}(t)$ and $s_{\mathcal{N}}(t)$ converge to $x_{\mathcal{B}}^{*}>0$ and $s_{\mathcal{N}}^{*}>0$ respectively, and therefore, remain bounded away from zero, we can conclude that $\hat{x}_{\mathcal{N}}(t)$ and $\hat{s}_{\mathcal{B}}(t)$ remain bounded.

Since the right-hand-side of (3.17) is bounded as $t$ tends to $\infty$, it is sufficient to show that $[x(t) \hat{s}(t)]^{T}[\hat{x}(t) s(t)]$ remains bounded below to conclude that both $x(t) \hat{s}(t)$ and $\hat{x}(t) s(t)$ have bounded norms as $t \rightarrow \infty$.

Let $v(t)=x^{\frac{1}{2}}(t) s^{\frac{1}{2}}(t)=w^{\frac{1}{2}}(t)$ and $\delta(t)=x^{\frac{1}{2}}(t) s^{-\frac{1}{2}}(t)$. Then,

$$
x(t) \hat{s}(t)=v(t) \delta(t) \hat{s}(t) \text { and } \hat{x}(t) s(t)=v(t) \delta^{-1}(t) \hat{x}(t) .
$$

Note that, $[\delta(t) \hat{s}(t)]^{T}\left[\delta^{-1}(t) \hat{x}(t)\right]=[\delta(t) \dot{s}(t)]^{T}\left[\delta^{-1}(t) \dot{x}(t)\right]=0$. Let $V(t)=\operatorname{diag}(v(t))$, $\Delta(t)=\operatorname{diag}(\delta(t))$, and $W_{0}=\operatorname{diag}\left(w_{0}\right)$. Then, $V^{2}(t)=X(t) S(t)=\frac{e^{T} w_{0}}{n}\left(e^{-(1-\beta) t}-e^{-t}\right) I+$ $e^{-t} W_{0}$. Now,

$$
\begin{gathered}
{[x(t) \hat{s}(t)]^{T}[\hat{x}(t) s(t)]=[v(t) \delta(t) \hat{s}(t)]^{T}\left[v(t) \delta^{-1}(t) \hat{x}(t)\right]} \\
=[\delta(t) \hat{s}(t)]^{T} V^{2}(t)\left[\delta^{-1}(t) \hat{x}(t)\right]
\end{gathered}
$$




$$
\begin{aligned}
= & e^{2(1-\beta) t}[\delta(t) \dot{s}(t)]^{T} V^{2}(t)\left[\delta^{-1}(t) \dot{x}(t)\right] \\
= & \frac{e^{T} w_{0}}{n}\left(e^{(1-\beta) t}-e^{(1-2 \beta) t}\right)[\delta(t) \dot{s}(t)]^{T}\left[\delta^{-1}(t) \dot{x}(t)\right] \\
& +e^{(1-2 \beta) t}[\delta(t) \dot{s}(t)]^{T} W_{0}\left[\delta^{-1}(t) \dot{x}(t)\right] \\
= & e^{-\beta t}\left[e^{(1-\beta) t / 2} \delta(t) \dot{s}(t)\right]^{T} W_{0}\left[e^{(1-\beta) t / 2} \delta^{-1}(t) \dot{x}(t)\right] .
\end{aligned}
$$

Recall from (3.9) that $\lim _{t \rightarrow \infty} \hat{w}_{j}(t)=\lim _{t \rightarrow \infty} e^{(1-\beta) t} w_{j}(t)=\frac{e^{T} w_{0}}{n}, \forall j$. Therefore, we have $\lim _{t \rightarrow \infty} \sqrt{\hat{w}_{j}(t)}=\lim _{t \rightarrow \infty} e^{(1-\beta) t / 2} v_{j}(t)=\sqrt{\frac{e^{T} w_{0}}{n}}$ and defining

$$
\tilde{v}(t)=e^{(1-\beta) t / 2}\left(v(t)-\frac{v(t)^{T} v(t)}{\rho} v^{-1}(t)\right),
$$

we have $\lim _{t \rightarrow \infty} \tilde{v}_{j}(t)=(1-\beta) \sqrt{\frac{e^{T} w_{0}}{n}}$.

Now, recalling equation (2.8) we observe that the vectors $e^{(1-\beta) t / 2} \delta^{-1}(t) \dot{x}(t)$ and $e^{(1-\beta) t / 2} \delta(t) \dot{s}(t)$ are orthogonal projections of the vector $-\tilde{v}(t)$ into the null space of $A \Delta(t)$ and range space of $[A \Delta(t)]^{T}$, respectively. Since we showed that the vector $\tilde{v}(t)$ is convergent as $t$ tends to $\infty$, both of these projections converge, and therefore, the expression in (3.18) converges to zero. Thus, $x(t) \hat{s}(t)$ and $\hat{x}(t) s(t)$ have bounded norms as $t \rightarrow \infty$.

It is interesting that the conclusion of the lemma above holds for any $\rho \geq 0$. An alternative proof of Lemma 3.3 can be obtained using the proof technique in [5]. Combining equation (3.1), Lemmas 3.2 and 3.3 we obtain the following result:

Lemma 3.4. Let $(\hat{x}(t), \hat{s}(t))$ be as in (3.16) and assume that $n<\rho \leq 2 n$. Then, $(\hat{x}(t), \hat{s}(t))$ remains bounded as $t$ tends to $\infty$.

Proof. From (3.1) we have that

$$
D_{\mathcal{B}}(t) \hat{x}_{\mathcal{B}}(t)=-\tilde{A}_{\mathcal{B}}^{+}(t) A_{\mathcal{N}} \hat{x}_{\mathcal{N}}(t)-\frac{n \cdot e^{(1-2 \beta) t}}{\rho\left(1-e^{-\beta t}\right)}\left(I-\tilde{A}_{\mathcal{B}}^{+}(t) \tilde{A}_{\mathcal{B}}(t)\right) u_{\mathcal{B}}(t)
$$

When, $\rho \leq 2 n$, the factor $\frac{n \cdot e^{(1-2 \beta) t}}{\rho\left(1-e^{-\beta t}\right)}$ is convergent as $t$ tends to $\infty$. Now, using Lemma 3.2 and the equations (3.11)-(3.12), we conclude that the second term in the righthand-side of the equation above remains bounded. Combining this observation with the the fact that $\hat{x}_{\mathcal{N}}(t)$ remains bounded as $t$ tends to $\infty$, we obtain that $D_{\mathcal{B}}(t) \hat{x}_{\mathcal{B}}(t)$ remains bounded. Using (3.10) we conclude that $\hat{x}_{\mathcal{B}}(t)$ is also bounded as $t$ tends to $\infty$. The fact that $\hat{s}(t)$ is bounded follows similarly. $\square$

Now, the following two results are easy to prove:

THEOREM 3.5. Let $(\hat{x}(t), \hat{s}(t))$ be as in (3.16). Then, we have that $\lim _{t \rightarrow \infty} \hat{x}_{\mathcal{N}}(t)$ and $\lim _{t \rightarrow \infty} \hat{s}_{\mathcal{B}}(t)$ exist and satisfy the following equations:

$$
\begin{aligned}
& \lim _{t \rightarrow \infty} \hat{x}_{\mathcal{N}}(t)=-\frac{e^{T} w_{0}}{n}(1-\beta)\left(s_{\mathcal{N}}^{*}\right)^{-1}, \\
& \lim _{t \rightarrow \infty} \hat{s}_{\mathcal{B}}(t)=-\frac{e^{T} w_{0}}{n}(1-\beta)\left(x_{\mathcal{B}}^{*}\right)^{-1} .
\end{aligned}
$$

Proof. From (3.17) we have that

$$
x_{\mathcal{B}}(t) \hat{s}_{\mathcal{B}}(t)+\hat{x}_{\mathcal{B}}(t) s_{\mathcal{B}}(t)=-\frac{e^{T} w_{0}}{n}(1-\beta) e_{\mathcal{B}}+e^{-\beta t}\left(\frac{e^{T} w_{0}}{n} e_{\mathcal{B}}-w_{\mathcal{B}}(0)\right) .
$$


Taking the limit on the right-hand-side as $t \rightarrow \infty$ we obtain $-\frac{e^{T} w_{0}}{n}(1-\beta) e_{\mathcal{B}}$. Since $s_{\mathcal{B}}(t) \rightarrow 0$ and $\hat{x}_{\mathcal{B}}(t)$ is bounded, we must then have that $x_{\mathcal{B}}(t) \hat{s}_{\mathcal{B}}(t)$ converges to $-\frac{e^{T} w_{0}}{n}(1-\beta) e_{\mathcal{B}}$. Since $x_{\mathcal{B}}(t) \rightarrow x_{\mathcal{B}}^{*}$, it follows that $\lim _{t \rightarrow \infty} \hat{s}_{\mathcal{B}}(t)$ exists and satisfies (3.21). The corresponding result for $\hat{x}_{\mathcal{N}}(t)$ follows identically.

Let

$$
\begin{gathered}
\xi_{\mathcal{B}}=X_{\mathcal{B}}^{*}\left(A_{\mathcal{B}} X_{\mathcal{B}}^{*}\right)^{+} A_{\mathcal{N}}\left(s_{\mathcal{N}}^{*}\right)^{-1}, \quad \sigma_{\mathcal{N}}=S_{\mathcal{N}}^{*}\left(G_{\mathcal{N}} S_{\mathcal{N}}^{*}\right)^{+} G_{\mathcal{B}}\left(x_{\mathcal{B}}^{*}\right)^{-1} \\
\pi_{\mathcal{B}}=X_{\mathcal{B}}^{*}\left(I-\left(A_{\mathcal{B}} X_{\mathcal{B}}^{*}\right)^{+} A_{\mathcal{B}} X_{\mathcal{B}}^{*}\right) w_{\mathcal{B}}(0), \quad \pi_{\mathcal{N}}=S_{\mathcal{N}}^{*}\left(I-\left(G_{\mathcal{N}} S_{\mathcal{N}}^{*}\right)^{+} G_{\mathcal{N}} S_{\mathcal{N}}^{*}\right) w_{\mathcal{N}}(0)
\end{gathered}
$$

Observe that $\pi_{\mathcal{B}}=0$ if and only if $w_{\mathcal{B}}(0) \in \mathcal{R}\left(X_{\mathcal{B}}^{*} A_{\mathcal{B}}^{T}\right)$ which holds, for example, when $\left(x_{0}, s_{0}\right)$ is on the central path and $w(0)=\mu e$ for some $\mu>0$-the observation that $e \in \mathcal{R}\left(X_{\mathcal{B}}^{*} A_{\mathcal{B}}^{T}\right)$ follows easily from the optimality of $x_{\mathcal{B}}^{*}$ for the first problem in (2.5). Similarly, $\pi_{\mathcal{N}}=0$ if and only if $w_{\mathcal{N}}(0) \in \mathcal{R}\left(S_{\mathcal{N}^{*}} G_{\mathcal{N}}^{T}\right)$.

THEOREM 3.6. Let $(\hat{x}(t), \hat{s}(t))$ be as in (3.16) and assume that $\rho \leq 2 n$. Then, we have that $\lim _{t \rightarrow \infty} \hat{x}_{\mathcal{B}}(t)$ and $\lim _{t \rightarrow \infty} \hat{s}_{\mathcal{N}}(t)$ exist. When $\rho<2 n$ we have the following identities:

$$
\begin{aligned}
\lim _{t \rightarrow \infty} \hat{x}_{\mathcal{B}}(t) & =\frac{e^{T} w_{0}}{n}(1-\beta) \xi_{\mathcal{B}}, \\
\lim _{t \rightarrow \infty} \hat{s}_{\mathcal{N}}(t) & =\frac{e^{T} w_{0}}{n}(1-\beta) \sigma_{\mathcal{N}} .
\end{aligned}
$$

When $\rho=2 n$, the following equations hold:

$$
\begin{aligned}
& \lim _{t \rightarrow \infty} \hat{x}_{\mathcal{B}}(t)=\frac{e^{T} w_{0}}{2 n} \xi_{\mathcal{B}}-\frac{n}{2\left(e^{T} w_{0}\right)} \pi_{\mathcal{B}}, \\
& \lim _{t \rightarrow \infty} \hat{s}_{\mathcal{N}}(t)=\frac{e^{T} w_{0}}{2 n} \sigma_{\mathcal{N}}-\frac{n}{2\left(e^{T} w_{0}\right)} \pi_{\mathcal{N}} .
\end{aligned}
$$

Proof. Recall equation (3.19). When $\rho<2 n$, the second term on the right-handside converges to zero since $e^{(1-2 \beta) t}$ tends to zero and everything else is bounded. Thus, using (3.10) and (3.11) we have $\lim _{t \rightarrow \infty} \hat{x}_{\mathcal{B}}(t)=-X_{\mathcal{B}}^{*}\left(A_{\mathcal{B}} X_{\mathcal{B}}^{*}\right)^{+} A_{\mathcal{N}} \lim _{t \rightarrow \infty} \hat{x}_{\mathcal{N}}(t)$ and (3.22) is obtained using Theorem 3.5. Similarly, one obtains (3.23).

When $\rho=2 n$, the factor in front of the second term in (3.19) converges to the positive constant $\beta=\frac{1}{2}$. Therefore, using Theorem 3.5 and equations (3.9)-(3.12) we get (3.24) and (3.25).

Limits of the normalized vectors $\left(\frac{\dot{x}(t)}{\|\dot{x}(t)\|}, \frac{\dot{s}(t)}{\|\dot{s}(t)\|}\right)$ are obtained immediately from Theorems 3.5 and 3.6:

Corollary 3.7. Let $(x(t), s(t))$ for $t \geq 0$ denote the solution of the ODE (2.9) with the initial condition $(x(0), s(0))=\left(x_{0}, s_{0}\right)$ with $\left(x^{0}, s^{0}\right) \in \mathcal{F}^{0}$ and assume that $\rho \leq 2 n$. All trajectories of this form satisfy the following equations:

$$
\lim _{t \rightarrow \infty} \frac{\dot{x}(t)}{\|\dot{x}(t)\|}=\frac{q_{P}}{\left\|q_{P}\right\|}, \quad \lim _{t \rightarrow \infty} \frac{\dot{s}(t)}{\|\dot{s}(t)\|}=\frac{q_{D}}{\left\|q_{D}\right\|}
$$

where

$$
\begin{gathered}
q_{P}=\left[\begin{array}{c}
\xi_{\mathcal{B}} \\
-\left(s_{\mathcal{N}}^{*}\right)^{-1}
\end{array}\right], \text { and } q_{D}=\left[\begin{array}{c}
-\left(x_{\mathcal{B}}^{*}\right)^{-1} \\
\sigma_{\mathcal{N}}
\end{array}\right], \quad \text { if } \rho<2 n, \\
q_{P}=\left[\begin{array}{c}
\xi_{\mathcal{B}}-\left(\frac{n}{e^{T} w_{0}}\right)^{2} \pi_{\mathcal{B}} \\
-\left(s_{\mathcal{N}}^{*}\right)^{-1}
\end{array}\right], \text { and } q_{D}=\left[\begin{array}{c}
-\left(x_{\mathcal{B}}^{*}\right)^{-1} \\
\sigma_{\mathcal{N}}-\left(\frac{n}{e^{T} w_{0}}\right)^{2} \pi_{\mathcal{N}}
\end{array}\right], \quad \text { if } \rho=2 n .
\end{gathered}
$$


When $\rho=2 n$ the TTY potential-function $\Phi_{\rho}(x, y)$ is a homogeneous function and $\exp \left\{\Phi_{\rho}(x, y)\right\}$ is a convex function for all $\rho \geq 2 n[17]$. The value $2 n$ also represents a threshold value for the convergence behavior of the KMY trajectories. When $\rho=2 n$ the direction of convergence depends on the initial point $\left(x^{0}, s^{0}\right) \in \mathcal{F}^{0}$ as indicated by the appearance of the $w_{0}=x^{0} s^{0}$ terms in the formulas. We note that, when $\rho<2 n$ the asymptotic direction of convergence does not depend on the initial point and is identical to that of the central path. Therefore, when $\rho<2 n$ all trajectories of the vector field given by the search direction of the Kojima-Mizuno-Yoshise's primaldual potential-reduction algorithm converge to the analytic center of the optimal face tangentially to the central path. We show below that the asymptotic behavior of the trajectories is significantly different when $\rho>2 n$ :

THEOREM 3.8. Let $(x(t), s(t))$ for $t \geq 0$ denote the solution of the ODE (2.9) with the initial condition $(x(0), s(0))=\left(x_{0}, s_{0}\right)$ and assume that $\rho>2 n$. Define

$$
\bar{x}(t)=e^{\beta t} \dot{x}(t), \text { and } \bar{s}(t)=e^{\beta t} \dot{s}(t) .
$$

If $\pi_{\mathcal{B}} \neq 0$ and $\pi_{\mathcal{N}} \neq 0$, then we have that $\lim _{t \rightarrow \infty} \bar{x}(t)$ and $\lim _{t \rightarrow \infty} \bar{s}(t)$ exist and satisfy the following equations:

$$
\lim _{t \rightarrow \infty} \frac{\bar{x}(t)}{\|\bar{x}(t)\|}=\frac{q_{P}}{\left\|q_{P}\right\|}, \text { and } \lim _{t \rightarrow \infty} \frac{\bar{s}(t)}{\|\bar{s}(t)\|}=\frac{q_{D}}{\left\|q_{D}\right\|},
$$

where

$$
q_{P}=\left[\begin{array}{c}
-\pi_{\mathcal{B}} \\
0_{\mathcal{N}}
\end{array}\right] \text {, and } q_{D}=\left[\begin{array}{c}
0_{\mathcal{B}} \\
-\pi_{\mathcal{N}}
\end{array}\right]
$$

Proof. From (3.1) we have that

$$
D_{\mathcal{B}}(t) \bar{x}_{\mathcal{B}}(t)=-\tilde{A}_{\mathcal{B}}^{+}(t) A_{\mathcal{N}} \bar{x}_{\mathcal{N}}(t)-\frac{n}{\rho\left(1-e^{-\beta t}\right)}\left(I-\tilde{A}_{\mathcal{B}}^{+}(t) \tilde{A}_{\mathcal{B}}(t)\right) u_{\mathcal{B}}(t)
$$

Note that, $\bar{x}_{N}(t)=e^{-(1-2 \beta) t} \hat{x}_{\mathcal{N}}(t)$. Since $\hat{x}_{\mathcal{N}}(t)$ is bounded and $-(1-2 \beta)<0$, we conclude that $\bar{x}_{N}(t) \rightarrow 0$. Therefore, using equations (3.30) and (3.9)-(3.12) we observe that $\bar{x}_{B}(t)$ converges to a positive multiple of $-\pi_{\mathcal{B}} \neq 0$ and immediately obtain the first equation in (3.29). The second identity in (3.29) is obtained similarly. 口

This final theorem indicates that when $\rho>2 n$, most trajectories associated with the Kojima-Mizuno-Yoshise algorithm converge to the analytic center of the optimal face tangentially to the optimal face and their direction of convergence depends on the initial point. In the exceptional case of $\pi_{\mathcal{B}}=0$ or $\pi_{\mathcal{N}}=0$ (for example, when $\left(x_{0}, s_{0}\right)$ is on the central path), the last term in (3.30) no longer dominates the righthand-side and in such cases we conjecture that the trajectory converges tangentially to the central path.

We conclude by noting the similarity of our asymptotic results to those of Monteiro [12]. In his analysis of the trajectories based on primal-only potential-reduction algorithms, Monteiro also finds that there is a threshold value of the potential function parameter that leads to different asymptotic behavior. In his case, this threshold value is $2|\mathcal{N}|$ rather than $2 n$, where $|\mathcal{N}|$ denotes the cardinality of the set $\mathcal{N}$ from the optimal partition of $\{1, \ldots, n\}$. Just like our case, when the potential function 
parameter is above this value, his trajectories converge tangentially to the central path and below the threshold the convergence is tangential to the optimal face.

\section{ACKNOWLEDGMENT}

The conclusion of Lemma 3.3 was stated without a proof in an earlier draft of this paper. The author would like to thank Margaréta Halická whose careful reading led to the correction of this omission. She also provided an alternative argument for the proof. The author would also like to thank an anonymous referee for bringing references $[7,9,10]$ to his attention and making several constructive comments. Results in these references helped make this a more concise paper.

\section{REFERENCES}

[1] I. Adler and R. D. C. Monteiro, "Limiting behavior of the affine scaling continuous trajectories for linear programming problems," Mathematical Programming, 50 (1991) pp. 29-51.

[2] K. M. Anstreicher, "Potential reduction algorithms," in: T. Terlaky, ed., Interior point methods of mathematical programming, (Kluwer Academic Publishers, Dordrecht, Netherlands, 1996) pp. 125-158.

[3] G. H. Golub and C. F. Van Loan, Matrix Computations, 2nd ed., (The Johns Hopkins University Press, Baltimore, 1989).

[4] O. Güler, "Limiting behavior of weighted central paths in linear programming," Mathematical Programming, 65 (1994) pp. 347-363.

[5] M. Halická, "Two simple proofs for analyticity of the central path," Operations Research Letters, 28 (2001) pp. 9-19.

[6] M. W. Hirsch and S. Smale, Differential equations, dynamical systems, and linear algebra (Academic Press, New York, 1974).

[7] M. Kojima, N. Megiddo, T. Noma, and A. Yoshise, A unified approach to interior point algorithms for linear complementarity problems, Vol. 538 of Lecture Notes in Computer Science, (Springer Verlag, Berlin, Germany, 1991).

[8] M. Kojima, S. Mizuno, and A. Yoshise, "An $O(\sqrt{n} L)$ iteration potential reduction algorithm for linear complementarity problems," Mathematical Programming, 50 (1991) pp. 331-342.

[9] L. McLinden, "An analogue of Moreau's proximation theorem, with applications to the nonlinear complementarity problem," Pacific Journal of Mathematics, 88 (1980) pp. 101-161.

[10] L. McLinden, "The complementarity problem for maximal monotone multifunctions," in: R. W. Cottle, F. Giannessi, and J.-L. Lions, eds., Variational Inequalities and Complementarity Problems, (Wiley, New York, 1980) pp. 251-270.

[11] R. D. C. Monteiro, "Convergence and boundary behavior of the projective scaling trajectories for linear programming," Mathematics of Operations Research, 16 (1991) pp. 842-858.

[12] R. D. C. Monteiro, "On the continuous trajectories for potential reduction algorithms for linear programming," Mathematics of Operations Research, 17 (1992) pp. 225-253.

[13] Yu. Nesterov, "Long-step strategies in interior-point primal-dual methods," Mathematical Programming, 76 (1997) pp. 47-94.

[14] K. Tanabe, "Centered Newton method for mathematical programming," in: M. Iri and K. Yajima, eds., Lecture Notes in Control and Information Sciences, (Springer-Verlag, Berlin, 1988) pp. 197-206.

[15] M. J. Todd, "Potential-reduction methods in mathematical programming," Mathematical Programming, 76 (1997) 3-45.

[16] M. J. Todd and Y. Ye, "A centered projective algorithm for linear programming," Mathematics of Operations Research, 15 (1990) 508-529.

[17] R. H. Tütüncü, "A primal-dual variant of the Iri-Imai algorithm for linear programming," Mathematics of Operations Research, 25 (2000),195-213.

[18] S. Wright, Primal-dual interior-point methods (SIAM, Philadelphia, 1997). 